\title{
AN ANALYSIS OF CHUN TAO IN THE SENSE OF SURVIVAL 浅析《春桃》中春桃的生存意识
}

\author{
Fu Ruomei \\ （付若玫） \\ Chinese Departement, Faculty of Languange and Culture, Binus University \\ Jl. Kemanggisan Ilir III no.45, Kemanggisan/ Palmerah, Jakarta Barat 11480 \\ rosemary@binus.edu
}

\begin{abstract}
In the novel "Chun Tao", Xu Dishan created Chun Tao as a new image of women, who has strong survival spirit, firm life philosophy and independent viability. Although Chun Tao's life was full of suffering, she never gave up hope. Through the analysis of the survival consciousness of Chun Tao revealed her spirits of independent and self-respecting, also showed the glory of humanity distributed by Chun Tao. Through close reading to analyze the character Chun Tao from the perspective of survival, intend to provide a new angle of observation for the reader to understand Chun Tao.

Keywords: Chun Tao, Survival, Humanity

\section{摘要}

许地山在《春桃》中塑造了一个新女性形象一一春桃。这是一个有着坚强的生存意念, 坚定的生存 理念, 具有独立生存能力的女性形象。虽然备受生活苦难折磨, 但依旧对生活充满希望。通过对春桃生 存意识的分析, 展现春桃所散发的独立自尊、圣洁仁爱的人性光辉。本文采用文本细读的方法从生存的 角度分析春桃这一人物形象, 希望能够为读者理解春桃提供一个新的观察角度。
\end{abstract}

关键词: 春桃，生存，人性 


\section{前言}

许地山的短篇小说《春桃》完成于 1934 年 3 月去印度考察的途中, 发表于《文学》第 3 卷第 1 期。《春桃》是许地山三十年代的杰出作品, 是许地山由浪漫主义向写实主义转变的 代表作品。许地山的作品大多有关宗教题材, 具有很强的宗教色彩。《春桃》几乎是他唯一没 有直接涉及宗教内容的作品, 但是, 在人物的命运和人物的精神性格上, 《春桃》还是体现了 作家悲悯仁爱的宗教情结。在《春桃》中, 许地山人道主义的思想得到充分体现, 树立了积极 进取、奋发有为的人生观。在创作方法上，人物、情节取之于现实生活，背景富于时代感。

三十年代的文坛, 受到五四新文化运动的影响, 出现了一大批反映女性寻求解放, 摆脱封 建束缚的文艺作品。《春桃》不同于当时流行的题材, 它写的不是知识女性追求爱情的浪漫故 事, 而是写一个社会底层的女性, 为了生存而同两个男人发生情感纠葛的故事。小说中春桃不 屈的生存意志, 积极的生活态度, 茂视礼法, 不畏流言, 勇于做自己的生存哲学令人肃然起 敬。春桃是在战乱年代中勇于追求自主和幸福的独立女性, 她坚守着自己的良心与良知, 维护 着自己的尊严和人格，展现了中国女性善良、坚韧的传统美德。

有关《春桃》的研究多集中在春桃、向高、李茂三人之间错综复杂的关系, 爱情、仁义如 何兼顾的矛盾冲突上。向高、李茂的选择是在城里向高是户主, 李茂是同居; 回到乡下, 李茂 是户主, 向高是同居, “你是咱们的媳妇。” 春桃给出的答案是, “你们都别想着咱们是丈夫 和媳妇”，“咱们三人就这样活下去”。逢增玉认为 “春桃那三人同居的决定，更是浪漫主义 的惊人之笔，使她的性格辉映出夺目的理想之光。” 王凯凯则认为三人最终的生活状态是 “在 经历了最初的笽尬与犹豫之后, 终于按照生活的需要接受了新的生活。”

春桃何以做出如此 “离经叛道” 的选择？春桃的选择基于她的生存理念，春桃具有强烈的 生存意识, 其对生存方式的选择, 坚定的生存理念, 对生命充满尊重与希冀, 这些都决定了三 人共同生活是春桃的必然选择。本文采用文本细读的方法从生存的角度分析春桃这一人物形 象, 希望能够为读者理解春桃提供一个新的观察角度。

\section{内容}

《春桃》写的是春桃与向高、李茂之间的情感纠葛。春桃本是财主的女儿, 成亲当天受乱 兵的冲击, 与丈夫李茂失散。后李茂参军, 双腿伤残后流落街头成了乞正。春桃流浪到北平以 捡破烂为生，与伙计向高日久生情，二人合作从事捡烂纸的营生，搭伙过日子。李茂巧遇春桃 后，被春桃带回住处。三人间剪不断，理还乱的感情纠葛构成了小说的最大冲突。

春桃与李茂是有龙风帖，喝过交杯酒的 “一夜夫妻” ; 与向高则是同住一铺炕，共同生活 了三年的 “事实夫妻”。注重名分的李茂、向高, 都希望能将春桃据为己有, 成为自己的媳 妇。春桃萵视名分, 认为那是有钱有势的人才在意的东西。春桃在意的是如何活下去, 如何有 尊严地活下去, 如何让三人都能更好地生活下去。春桃最终选择了两个都不放弃, 三人共同生 活。春桃对生命的尊重, 面对苦难的从容, 对向高的依恋与对李茂的情义凸显了她心灵的纯 净，散发出圣洁的人性之光。 


\section{春桃具有强烈的生存愿望}

春桃出身财主家庭, 相对普通农民其生活自然会舒适安逸得多。听从父母之命嫁给自家的 帮工, 也是出于生存的考量。“李茂告诉向高, 春桃的父亲是个乡下财主, 有一顷田。他自己 的父亲就在他家做活和赶叫驴。因为他能瞄很准的枪, 她父亲怕他当兵去, 便把女儿许给他, 为的是要他保护庄里的人们。” 如果没有兵乱也许就此生活下去。但春桃的命运注定是坎坷 的。兵乱与新婚丈夫失散, 春桃自此独自踏上逃荒之路。

逃到北京的春桃努力挣扎，自谋生路。作者在此处的处理颇耐人寻味。一般与家人失散的 独身女子, 会尽力寻找失散的亲人, 以求早日团聚, 有所依靠。春桃逃荒跑到北京, 如何生存 下去是春桃面临的首要问题。春桃对此有十分明确的想法, 解决生存问题必须依靠自己。她出 去帮佣, 捡废纸; 却从未想过寻找离散的丈夫; 更没有打算回乡下寻亲, 以求庇护。由此可见 春桃已经具有强烈的独立意识。逃荒途中首遇向高, 二人虽然同行了几百里, 但最终向高选择 去投亲靠友, 春桃却选择了踏上遥远陌生的进城之路。二人的不同选择更突显了春桃的独立自 强。

当李茂不堪忍受乞讨之苦表示: “若是这样下去, 我可受不了, 非上吊不可。” 春桃湿了 眼眶, 但还是静默着, 没有对李茂的想法发表议论。但从后来的对话中却可以看出春桃并不赞 同李茂意图轻生的念头。李茂看到春桃的处境不错, 不由感叹: “春桃, 你这几年呢? 这小小 地方虽不如咱们乡下那么宽敞, 看來你倒不十分苦。” 春桃回应: “谁不受苦? 苦也得想法子 活。在阎罗殿前, 难道就燋不不见笑脸? ” 这就是春桃的生存宣言。任命运百般为难, 任造化 无情弄人，春桃选择勇敢乐观地面对，坚强地活下去。

\section{春桃忠于内心的生存原则}

春桃在京城找到了营生, 除了保证自己的生活, 甚至还帮助向高、李茂生存。生活上的变 故, 春桃坦然接受, 没有李茂、向高对以往生活的留恋, 拥有的是适应环境, 坚定走下去的决 心。如果说春桃的一切所为只是为了生存, 那就市负了许地山在春桃塑造中的良苦用心。春桃 不止是要生存下去, 而且要追随自己的内心, 有尊严地, 自在地活下去。春桃逃荒到北京, 许 地山安排了好几条生存之路供其选择。

春桃容貌秀丽。“在破草帽底下虽看不清她的脸, 当她与卖酸梅汤的打招呼时, 却可以理 会她有滿口雪白的牙齿。” “她换了一条蓝布裤。上身是白的, 脸上虽沒脂粉, 却呈露着天然 的秀丽。”许地山也感叹, “春桃的模样, 若脱去破帽子, 不用说到瑞蚗祥或別的上海成衣 店, 只到天桥搜罗一身落伍的旗袍穿上, 坐在任何草地, 也与 “还是他好” 里那摩登女差不上 下。” 拥有出众外貌的春桃并不愿意靠容貌谋生。她不肯再嫁, 虽然 “若她肯嫁的话, 按媒人 的行情, 说是二十三四的小寡妇, 最少还可以值得一百八十的。” 她对以色事人更是充满鄙 夷。“別糟蹋我的晚香玉。晚上戴花, 又不是窑姐儿。”为了生存, 老舍笔下的月牙儿选择 了卖身, 春桃虽有秀丽的容貌, 她选择了将美丽的容颜掩藏在破烂的草帽低下。沉重肮脏的垃 圾管压弯了她的腰, 但她的人格是挺立的, 她的尊严是不屈的。

春桃尝试做过女佣, 在西洋人家上工, 并深得主妇喜爱。按说这是条不错的谋生之路, 对 逃荒在外的乡下姑娘而言简直是美差。就是这样的美差, 春桃却主动辞工了。辞工的原因是受 不了主人家的饮食习惯。“她见主人老是吃牛肉, 在馒头上涂牛油, 喝茶还要加牛奶, 来去鼓 着一阵臊味, 闻不惯。有一天, 主人叫她带孩子到三贝子花园去, 她理会主人家的气味有点像 从虎狼栏里发出来的, 心里越发难过, 不到两个月, 便辞了工。” 春桃的生存哲学不只为肉体 
的存活, 她更珍视的是内心的安宁与纯净。杀生为佛法大忌, 春桃的慈悲之心与宗教悲悯情怀 由此可见一斑。“到平常人家去, 乡下人不惯当差, 又挨不得骂, 上工不久, 又不干了。”春 桃自尊心强, 追求自由独立, 不愿受控于人的个性在此处也得以体现。

最后 “在穷途上, 她自己选了这捡烂纸换取灯儿的职业, 一天的生活, 勉強可以维持下 去。” 春桃虽然 “整天在街头巷尾垃圾堆里讨生活……, 一天到晚在烈日冷风里吃尘土, 可是 生来爱干净, 无论冬夏, 每天回家, 她总得净身洗脸。” 春桃的净身洗浴在小说中多次出现。 逢增玉在《乱世尘缘中的超俗入圣一一许地山小说《春桃》新解》中曾对春桃的 “尚洁” 情结 做过深入分析, 认为 “春桃是立足于尘世泥土上、充满人间气息和佛道色彩的 “北方民间女 神'。”

春桃最终选择的是身体上最苦、最累、最脏的职业, 但她由此却保有了内心的轻松、坦然 与洁净。

\section{春桃具有极强的生存能力}

春桃具有极强的生存能力。第二次与向高相遇, 向高仅靠给人帮工维持朝不保夕的生活, 而此时的春桃已经可以自食其力，且 “事业渐次发达了。” 同是逃难之人，向高是男人，又是 高小毕业生, 本应更容易谋生, 但他的生活居然不如春桃。后来还是春桃收留了他给自己当伙 计。春桃的生存能力之强不得不令人佩服。

春桃不畏劳苦。捡烂纸换取灯儿的职业是极其辛苦的, “她背上担负得很重, 甚至不能把 腰挺直, 只如骆驼一样, 庄严地一步一步踱到自己门口。” “一天到晚在烈日冷风里吃尘 土。” “她自己干那吃力的工作, 除去下大雨以外, 在狂风烈日底下, 是一样地出去捡货。尤 其是在天气不好的时候, 她更要工作, 因为同业们有些就不出去。”

春桃具有经营意识。瓜棚低下的 “买卖经” 就是春桃对一日工作的总结与对未来工作的规 划。“若是多个人在家替你归着东西, 你白天便可以出去摆地摊, 省得货过别人手里, 卖漏 了。”对自己在城里的生计发展也有着明确的规划。“这年头, 那一个乡下都是一样, 不闹 兵，便闹贼；不闹贼，便闹日本，谁敢回去？还是在这里捡捡烂纸罢。” “若是每天在烂纸 堆里能检出八毛钱就算顶不错, 还用回乡下种田去? 那不是自找罪受么? ’ 春桃愉悦的声音就 像春深的莺啼一样。”

对捡破纸事业的了解与思考, 对前景发展的规划决定了春桃绝对的领导地位。没有春桃, 三人捡纸片的生意是维持不下去的。正由于对生存困境有着清晰地思考, 所以春桃作出了三人 共同生活的大胆选择。“她心中已经派定李茂在家把旧邮票和纸烟盒里的画片检出来。那事 情, 只要有手有眼, 便可以做。她合一合, 若是天天有一百几十张卷烟画片可以从烂纸堆里检 出来, 李茂每月的伙食便有了门。” “至于向高还是让他检名人书札, 或比较可以多卖钱的东 西。”春桃对三人共同生活充满信心。“近来我常想着得多找一个人来帮忙，可巧茂哥来了。 他不能走动, 正好在家管管事, 检检纸。你当跑外卖货。我还是当捡货的。咱们三人开公 司。”

正因为经济的独立，使得春桃有足够的勇气与实力对抗两个男人的夫权统治。李茂在意名 分, 怕当王八。春桃当即反驳: “有钱有势的人才怕当王八。像你, 谁认得? 活不留名, 死不 留姓, 王八不王八, 有什么相干? 现在, 我是我自己, 我做的事, 决不会玷着你。”一个独自 自强、不畏人言的女性形象跃然纸上。面对蜚短流长, 春桃秉持的生存哲学是 “ “人打还打, 
人骂还骂, 的态度。不是个弱者, 不打骂人, 也不受人打骂。我们听她教训向高的话, 便可以 知道。‘若是人笑话你，你不会揍他？你露什么怯？咱们的事，谁也管不了。

春桃选择了三人一同生活, 虽然开始受到李茂、向高的强烈反对。二人为互相成全, 一个 选择自我毁灭, 另一个选择离家出走。面临李茂自杀, 向高出走, 春桃选择的排解心中苦闷的 方式是 “她仍旧把破帽戴着, 背上管子。” 因为 “作活是她的天性, 虽在沉闷的心境中, 她也 要干。”最终二人还是回到了春桃身边，按照春桃设计的生活方式相处下去。春桃的选择最根 本的原因是对生命的尊重, 对生存的渴望, 对尊严与道义的坚守。

\section{结语}

我该如何存在？春桃的选择是：追随自己的内心, 以自己选择的方式存在。“你们都别想 着咱们是丈夫和媳妇” “咱们三人就这样活下去。”许地山在形容春桃向高二人的生活状态时 写道: “他们同居这些年, 生活状态, 若不配说像鸳莺, 便说像一对小家雀吧。”只羡驾莺不 羡仙, 那是在吃饱喝足后发出的感慨。春桃与向高是苦苦挣扎在生死线上的人，用一对忙忙碌 碌，为生计奔波的“小家雀”来形容二人关系再贴切不过。这也回归到了生存的主题。

春桃所关注的最根本的问题就是生存。为了生存可以吃苦受累, 满身污浊, 但内心的纯 净, 自由却不可放弃, 不容侵犯。依照春桃的生存哲学, 只要活下去, 可以不必在意别人的眼 光。但生存并不意味着只是活下去。做人的尊严，对生命的慈悲，情义仁爱都是不可舍弃的。 在兵荒马乱的年月、在动荡的社会中，春桃即使孤身一人仍然顽强地生存了下来，每天早出晚 归的勤奋工作使得自己能够获得经济独立。她选择了一条虽不为传统礼教所容, 但却可以兼顾 爱情与道义的三人共同生活的道路。春桃是美善的极至，她最宝贵之处在于无论遭遇多少苦难 仍旧保有一颗仁爱之心。在苦难中，春桃彰现了人性的尊严。

\section{参考文献}

胡山林. 文学欣赏. 北京:清华大学出版社，2008

许地山. 春桃. 中国现代文学馆 编. 北京:华夏出版社, 2008

逢增玉. 乱世尘缘中的超俗入圣一一许地山小说《春桃》新解. 名作欣赏, 2010 年第 9 期 王凯凯. 生存意义下的价值选择一一浅析许地山小说《春桃》. 文艺生活: 中旬刊, 2010 年第 5 期 朱银平. 生存困境的哲理性表达一一论许地山作品《春桃》.南方论刊, 2010 年第 1 期。 